\title{
Study on Dynamic Design Technique of Machine Tools Structure Based on Energy Balance
}

\author{
Guangchen $\mathrm{Xu}^{1}{ }^{1}$, Nannan Zhang ${ }^{2}$, Zeyu Weng ${ }^{2}$ \\ ${ }^{1}$ Ying Kou Institute of technology, Ying Kou, 115000, China \\ ${ }^{2}$ Key Laboratory of Special Purpose Equipment and Advanced Manufactory Technology, Ministry of \\ education, Hangzhou, 310014, China
}

Keywords: machine tools structure; dynamic design; energy balance; finite element technique.

\begin{abstract}
The energy balance of system is one of the basis for the dynamic design of machine structure. The paper applied finite element technique to machine dynamic design based on the energy balance, in view of finite element model of machine dynamics structure, mean squared deviations of various energy distribution were put forward to quantitatively describe the structural dynamic characteristics, and the technique was applied to the structural dynamic characteristics analysis of a surface grinder, the weak link of the grinding machine structure was found, which had instruction function for structure optimization of the grinding machine. The result proved this structure dynamics design technique had obviously superiority.
\end{abstract}

\section{Introduction}

The machine tools is the most basic equipment in the machine manufacture industry, along with the development of the high-speed and precise maching technology, the requestment of dynamic performance of machine tools becomes higher and higher. Under the known working condition of the machine tools, the dynamic design of the machine tools is a process to seek for a perfect machine tool structure which satisfy its predetermined dynamic property. G.Bianchi and other scholars ${ }^{[1]}$ of American Catholic University cimbined dynamic design of the machine tools and control to carry on dynamic optimization design, J.M. Vance of Lowa State University, T.P. Yep of ISU Research center and other scholars ${ }^{[2]}$ applied the virtual reality technology to carry on the shape optimization design of machine structure. X.L. Zhang and Y.S. Xu ${ }^{[3]}$ of Tianjin University utilized structural dynamic design principles and variable parsing technique of finite element method to propose a dynamic design method and the flow of the bed structure of numerical control machine. Z.H. Yin and B.Y. Liao ${ }^{[4]}$ of Kunming University of Science and Technology minutely discussed the basic process of using the optimal criterion to optimize the mechanical performance of grinder main axle system, and discussed superiority of optimization criterion to carry on optimal design of mechanical structures.

Research on theory of the optimization based on energy balance studies was obtained certain application, and some progress had been made. M. Yoshimura, T. Hoshi and other scholars ${ }^{[5-}$ ${ }^{7]}$ detailed introduced the dynamic optimal design principle and method, which took the most small danger of regeneration flutter as a goal and was in the basis of modal flexibility and energy balance. Z.L. Zhang ${ }^{[8]}$ of machine tool staffroom of Harbin Industry University applied energy balance principle to optimize the machine tools, the lumped mass model of projection grinder was established to conduct the dynamic characteristic research, the mathematics model was applied to carry on the theoretical analysis and the computation of machine tools, then the dangerous mode and link were pointed out, and the improved suggestions were put forward for the weak parts. W. Z. Zheng ${ }^{[9]}$ of Mechanical and electrical Branch school of Shanghai Jiao Tong University used the energy balance principle for the optimization, The lumped mass model was adopted to carry on the energy distribution computation of the wheel head of multi-purpose cylindrical grinder M1432A, then the weak part of the complete machine was discovered, which provided theoretical basis for the following vibration-proof optimization design of the wheel head. W.L. Xiong and other scholars ${ }^{[10]}$ of Hunan University, in the study of dynamic optimization design of high speed and 
high precision spindle unit, the lumped mass model was established, the combining principle of modal flexibility and the energy balance was adopt to distinguish the weak link of the electrical spindle, then the structural design parameters of these weak links were changed under a certain condition to reduce the modal flexibility and raise the optimized efficiency. Known from existing documents, the domestic and foreign scholars adopted lumped mass model as the application of optimize theory based on energy balance for the analysis and optimization.

In the mechanical analysis and the dynamic design of machine tools, most common modle includes: lumped parameter model, distributed mass model and finite element model. In 1964, Malitback and Toylor and Tobias proposed a method with two simple unit to connect lumped mass to establish dynamics model; Afterward M. yoshimura and T. Hoshi established distributed mass dynamics model of the planing milling machine with single column. During 1970-1971 years, international production engineering research association CIRP had conducted the union research on distributed mass beam and lumped mass for establishing the machine dynamics model. In order to enhance analysis and design precision, the model of the closer actual structure was used to conducts machine dynamics research, some scholars brought the finite element method into dynamic characteristic research of machine tools. In 1968, Stephan applied the finite element in the modelling of machine structure ${ }^{[11]}$. The 1990s, along with the development of computer technology, finite element method became a most widely used modeling method in the dynamic analysis of complex mechanical structure and dynamic design.

This paper intended to take the finite element model as the foundation to apply optimization method based on energy balance. Through the energy analysis, structural dynamic characteristics evaluation and modification were carrried out to achieve structural optimization. The author hope that makes the optimization method based on energy balance more effective and convenient in practice.

\section{Energy Calculation and Evaluation of the Structural Dynamic Characteristics}

The total energy of the whole structure in vibration process is the synthesis of the substructure energy. Established the maximum inertia energy of a substructure $s$ is $T_{s r}$ when vibration mode is the $r$ order, and maximum elastic energy is $V_{s r}$. Since energy is a scalar, maximum inertial energy of the entire structure is $T_{A r}$ for the r-order mode vibration, and its maximum elastic energy is $V_{A r}$, which is respectively corresponding energy synthesis of all substructures, that are:

$$
\begin{aligned}
& T_{A r}=\sum_{s=1}^{N} T_{s r} \\
& V_{A r}=\sum_{s=1}^{N} V_{s r}
\end{aligned}
$$

Various types of energy of substructure could respectively represent:

$$
T_{s r}=\frac{1}{2} \omega_{n r}^{2}\left\{A^{(r)}\right\}_{s}^{T}[m]_{s}\left\{A^{(r)}\right\}_{s} \quad \text { (3) } V_{s r}=\frac{1}{2}\left\{A^{(r)}\right\}_{s}^{T}[k]_{s}\left\{A^{(r)}\right\}_{s}
$$

Where $\left\{A^{(r)}\right\}_{s}$ is the amplitude matrixt of all nodes of the unit $s$ in the direction of the moving coordinate when the system is the r-order mode vibration mode; $\left\{A^{(r)}\right\}_{r}^{T}$ is the transposing matrix of $\left\{A^{(r)}\right\}_{s} ;[m]_{s}$ is the inertia matrix of unit $s ;[k]_{s}$ is the stiffness matrix of unit $s$.

The ratio of various types of energy of substructure and corresponding energy of the entire structure could define inertia distribution rate and elastic energy distribution rate of the r-order mode of the substructure s. The substructure with higher energy distribution rate indicates its quality is too large or its stiffness is too low, this substructure should be improved. A substructure with higher inertia energy distribution rate should focus on reducing its weight, with higher elastic energy distribution rate should emphasize to raise its stiffness, which makes energy distribution rate 
of the substructure tend to well-distributed, this is the machine structure optimization theory based on the energy balance.

Regarding the estimate of machine energy distribution, mean square deviation of each mode kinetic and potential distribution of vibration system could be drawn to quantitatively describe the uniformity of each mode energy distribution of machine structure, and to determine the degree of its deviation from optimum design.

The mean square deviation of each mode kinetic and potential distribution of machine tool vibration system could respresent as follows:

$$
\begin{gathered}
\sigma_{r T}=\sqrt{\frac{\sum_{i=1}^{n}\left(T_{i}-\bar{T}\right)^{2}}{n}} \\
\sigma_{r V}=\sqrt{\frac{\sum_{i=1}^{n}\left(V_{i}-\bar{V}\right)^{2}}{n}}
\end{gathered}
$$

Where: $\sigma_{r T}$ is the mean square deviation of the r-order kinetic energy, Ti is the value of kinetic energy of unit $\mathrm{i}, \bar{T}$ is the average value of the r-order kinetic energy, $\sigma_{r T}$ is the the mean square deviation of the r-order potential energy, $V_{i}$ is the value of potential energy of unit $\mathrm{i}, \bar{V}$ is the average value of the r-order potential energy, $i$ is the unit $i, n$ is the unit number.

Obviously, if lumped parameter model of improved structure was established for further analysis, there would be very tedious to estimate related parameter of the model in the modelling process, especially in order to increase the model precision, while the number of degrees of freedom was increased. In the process of structure dynamics modelling, structure modification and computation of the energy distribution rate, the superiority of the application of finite element model was fully demonstrated. Known from existing literature, the domestic and foreign scholars were mainly aimed at lumped parameter model to carry on structure optimization based on energy balance, while structure optimization of the finite element model was not involved.

\section{Structure Dynamics Analysis of Machine Tools Based on Energy Balance}

The application of the finite element technology in structural dynamic characteristics analysis is an effective and convenient method, here a certain ultra-precise surface grinding machine adopted theis method to analyse its dynamic characteristics.

The finite element modal analysis and the energy computation was carried on with the ANSYS software. Natural frequency of its first six order modal respectively are $51.8 \mathrm{~Hz}, 88.7 \mathrm{~Hz}, 119.1 \mathrm{~Hz}$, $155.9 \mathrm{~Hz}, 171.1 \mathrm{~Hz}$ and $231.26 \mathrm{~Hz}$.

The energy distributed situation of kinetic energy, potential energy of the first six order modal is shown in Fig. 1 and Fig. 2:

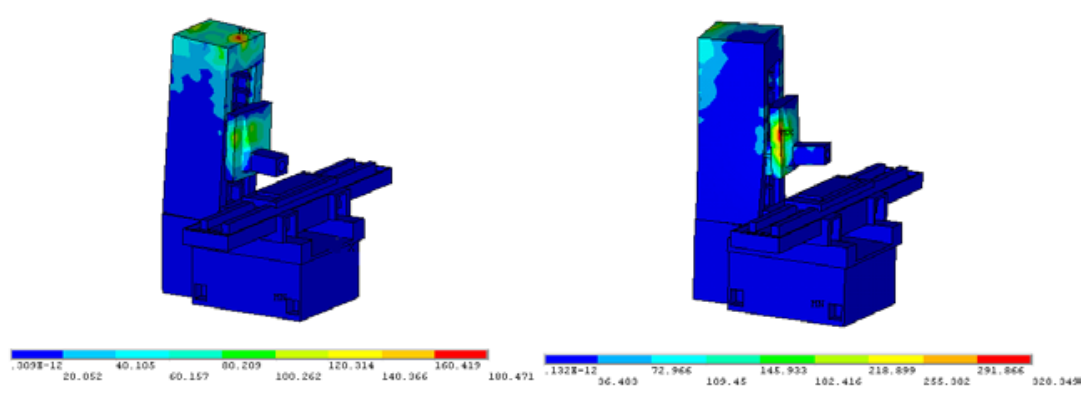

(a)The fisrt order

(b)The second order 


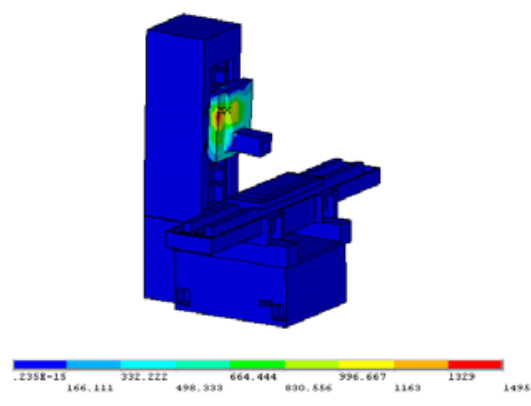

(c) The third order

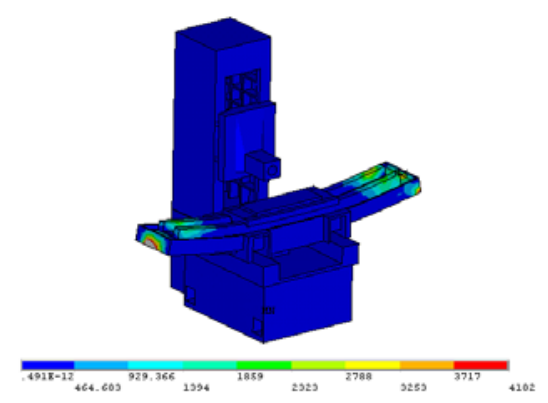

(e)The fifth order

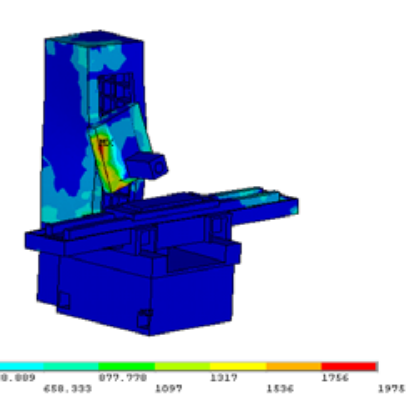

(d)The fourth order

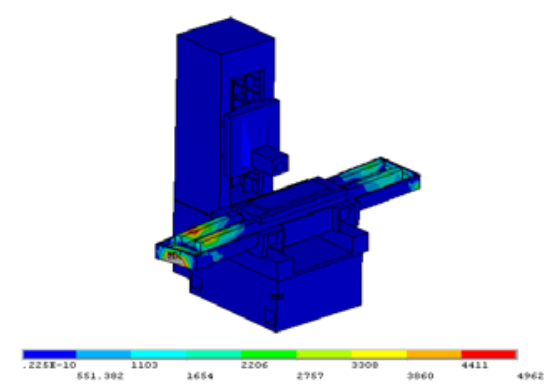

(f)The sixth order

Fig. 1 Modal kinetic energy distribution of each order of ultra-precise surface grinding machine

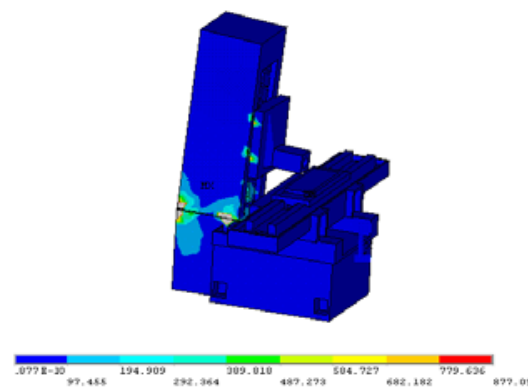

(a)The first order

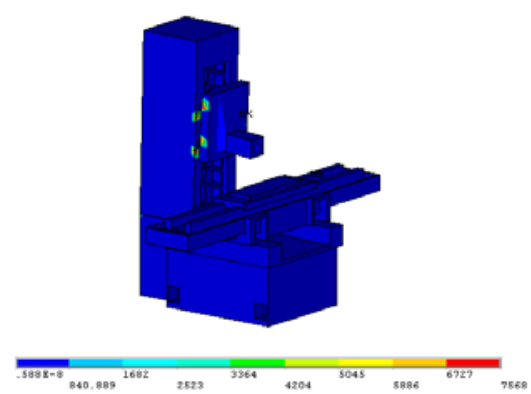

(c) The third order

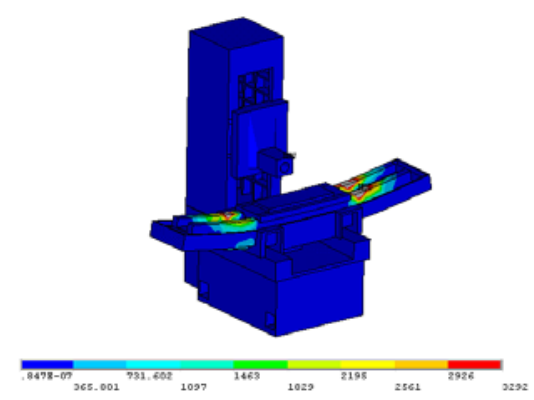

(e)The fifth order

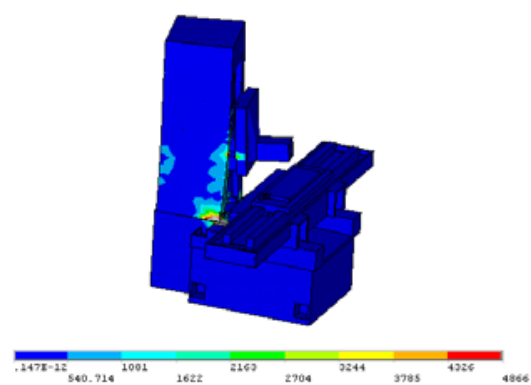

(b)The second order

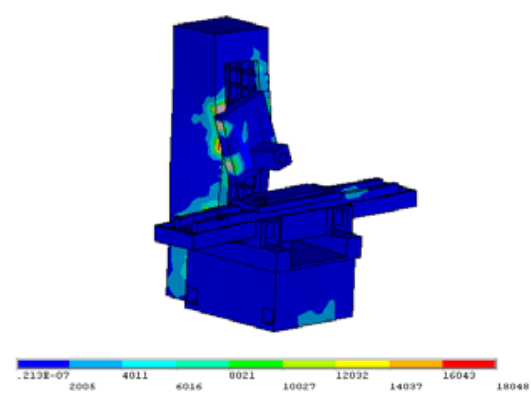

(d)The fourth order

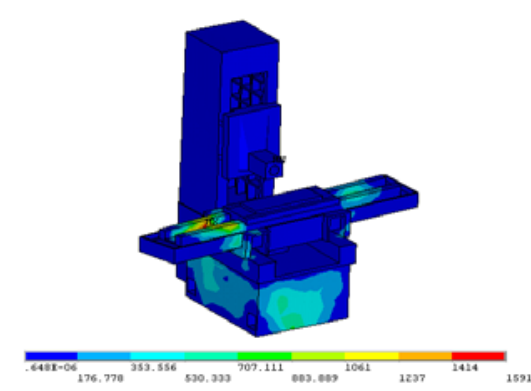

(f)The sixth order

Fig. 2 Modal potential energy distribution of each order of ultra precise surface grinding machine 
The mean squared deviation of kinetic energy and potential energy of the first six step modality is shown in Table 1, and the mean squared deviation in Table 1 illustrates the degree of uniformity of kinetic energy and potential energy distribution is lower.

Known from the kinetic energy distribution, concentrated places of kinetic energy are mainly the grinding head, the upper and lower ends of column, the both ends of extension units and the front bed board.

Through the analysis, the mainly reasons are obtained as follows:

(1) The kinetic energy of wheelhead is big, the reason is possibly because the wheelhead has large mass, or the stiffness of wheelhead-column bonding surface is small, which causes the amplitude of the wheelhead to be large, thus its kinetic energy is increased;

Table 1 Modal kinetic energy and potential energy of machine first six step modals

\begin{tabular}{|c|c|c|c|c|c|c|}
\hline Square deviation $\sigma(\mathrm{J})$ & $\begin{array}{c}\text { The first } \\
\text { order }\end{array}$ & $\begin{array}{c}\text { The second } \\
\text { order }\end{array}$ & $\begin{array}{c}\text { The third } \\
\text { order }\end{array}$ & $\begin{array}{c}\text { The fourth } \\
\text { order }\end{array}$ & $\begin{array}{c}\text { The fifth } \\
\text { order }\end{array}$ & $\begin{array}{c}\text { The sixth } \\
\text { order }\end{array}$ \\
\hline $\begin{array}{c}\text { Mean squared deviation of } \\
\text { kinetic energy } \sigma_{r T}(\mathrm{~J})\end{array}$ & 2.4 & 7.4 & 19.6 & 53.7 & 76.8 & 93.2 \\
\hline $\begin{array}{c}\text { Mean squared deviation of } \\
\text { potential energy } \sigma_{r V}(\mathrm{~J})\end{array}$ & 30.6 & 60.6 & 191.4 & 461.6 & 77.2 & 65.1 \\
\hline
\end{tabular}

(2) Kinetic energy distribution of column is centralized, for the longitudinal of column is small, and the both ends has large mass, which makes amplitude of the ends of column, especially the upper column become large, then appears kinetic energy of the column ends concentrated;

(3) The carriage above joint surface of carriage-bed has low stiffness, and the both carriage ends has big mass, thus the amplitude of both carriage ends increases big, and kinetic energy is larger, while front plane of bed has little influence on machining precision of machine tools.

Known from the potential energy distribution, potential energy is mainly concentrated at the joint surface of grinding-column, the carriage above carriage-column and the front board of bed.

The following main reasons are obtained after the analysis:

(1) The stiffness of joint surfaces of grinding-column is low, which could easily cause potential energy concentrated;

(2) Because the both ends of carriage is much longer, then less stiffness is caused, which could easily produces bigger deformation.

The above analysis result that structure dynamics analysis of machine tools base on energy balance may directly guide improve mental design of machine tools.

\section{Conclusions}

The energy balance is one of important theories of machine structure dynamics design, the application of the finite element technology in machine structure dynamics analysis based on the energy balance has fully demonstrated its superiority in the process of the structure dynamics modelling, the structure modification and computation of the energy distribution rate. Firstly, the process of dynamics modelling could rely on the software, and that has enough high precision; Secondly, structure energy distribution situation and mode shape could be directly checked, which is conveniently to use the weak link analysis method based on energy balance and mode shape as a supplement to carried on the structure improvement, and forecast the improvement effect.

\section{References}

[1] G. Bianchi, F. Daolucci: submitted to Annals of the CIRR (1996)

[2] J.M.Vance, T.P.Yep: submitted to Structual Incoporting Joint Dynamincs Data(1979)

[3] X.L. Zhang, Y.S. Xu and W.H. Zhong: submitted to Journal of Mechanical Strength (2005) 
[4] Z.H. Yin, X.M. Zhou and B.Y. Liao: submitted to Journal of Kunming University of Science and Technology (2001)

[5] M.Yoshimura, T.Hoshi: submitted to 12th M.T.D.R. (1971)

[6] Tetutaro Hoshi and Masataka Yoshimura: submitted to 14th M.T.D.R. (1973)

[7] Mosataka, Yoshimura, submitted to 16th M.T.D.R. (1975)

[8] Z.L. Zhang: submitted to Journal of Harbin Institute of Technology (1987)

[9] W.Z. Zheng: submitted to Machine (1982)

[10] W.L. Xiong, J.L. Wen and H.W. Huang: submitted to Journal of Machine Design (2004)

[11] Z.F. Liang: Master paper, Kunming University of Science and Technology (2003) 